\title{
On Signed Product Cordial Labeling
}

\author{
Jayapal Baskar Babujee, Shobana Loganathan \\ Department of Mathematics, Anna University, Chennai, India \\ E-mail: \{baskarbabujee, shobana_2210\}@yahoo.com \\ Received September 19, 2011; revised October 25, 2011; accepted November 3, 2011
}

\begin{abstract}
A new concept of labeling called the signed product cordial labeling is introduced and investigated for path graph, cycle graphs, star- $K_{1, n}$, Bistar- $B_{n, n}, P_{n}^{+}, n \geq 3$ and $C_{n}^{+}, n \geq 3$. Some general results on signed product cordial labeling are studied.
\end{abstract}

Keywords: Graph, Labeling, Function, Cordial Labeling

\section{Introduction}

If the vertices of the graph are assigned values subject to certain conditions then it is known as graph labeling. Most of the graph labeling problems have the following three common characteristics: a set of numbers for assignment of vertex labels, a rule that assigns a label to each edge and some condition(s) that these labels must satisfy.

Cordial labelings were introduced by Cahit [1] who called a graph $G$ cordial if there is a vertex labeling $f: V(G) \rightarrow\{0,1\}$ such that the induced labeling

$f^{*}: E(G) \rightarrow\{0,1\}$, defined by $f^{*}(x y)=|f(x)-f(y)|$, for all edges $x y \in E(G)$ and with the following inequalities holding: $\left|v_{f}(0)-v_{f}(1)\right| \leq 1$ and

$\left|e_{f}(0)-e_{f}(1)\right| \leq 1$, where $v_{f}(i)$ (respectively $e_{f}(i)$ ) is the number of vertices (respectively, edges) labeled with $i$. Sundaram and Somasundaram [2] introduced the notion of product cordial labelings. A product cordial labeling of a graph $G$ with vertex set $V$ is a function $f$ from $V$ to $\{0,1\}$ such that if each edge $u v$ is assigned the label $f(u) f(v)$, the number of vertices labeled with 0 and the number of vertices labeled with 1 differ by at most 1 , and the number of edges labeled with 0 and the number of edges labeled with 1 differ by at most 1. A graph with a product cordial labeling is called a product cordial graph.

For detail survey on graph labeling one can refer Gallian [3]. Let $G=(V, E)$ be a graph. As given in [4] a mapping $f: V(G) \rightarrow\{0,1\}$ is called binary vertex labeling of $G$ and $f(v)$ is called the label of the vertex $v$ of $G$ under $f$. For an edge $e=u v$, the induced edge labeling $f^{*}: E(G) \rightarrow\{0,1\}$ is given by $f^{*}(e)=|f(u)-f(v)|$. Let $v_{f}(0), v_{f}(1)$ be the number of vertices of $G$ having labels 0 and 1 respectively under $f$ and let $e_{f}(0), e_{f}(1)$ be the number of edges having labels 0 and 1 respectively under $f^{*}$. A binary vertex labeling of a graph $\mathrm{G}$ is called a cordial labeling if $\left|v_{f}(0)-v_{f}(1)\right| \leq 1$ and $\left|e_{f}(0)-e_{f}(1)\right| \leq 1$.

In Section 2, we introduce the definition of signed product cordial labeling and work for few fundamental graphs. In Section 3 we prove that $P_{n}^{+}, C_{n}^{+}$and bistar graph $B_{n, n}$ are signed product cordial. Finally in Section 4 , we prove the existence of signed product cordial labeling for some general graphs.

\section{Signed Product Cordial Labeling}

We now introduce the definition of signed product cordial labeling.

Definition 2.1: A vertex labeling of graph $G$

$f: V(G) \rightarrow\{-1,1\}$ with induced edge labeling

$f^{*}: E(G) \rightarrow\{-1,1\}$ defined by $f^{*}(u v)=f(u) f(v)$ is called a signed product cordial labeling if

$\left|v_{f}(-1)-v_{f}(1)\right| \leq 1$ and $\left|e_{f}(-1)-e_{f^{*}}(1)\right| \leq 1$, where

$v_{f}(-1)$ is the number of vertices labeled with $-1, v_{f}(1)$ is the number of vertices labeled with $1, e_{f^{*}}(-1)$ is the number of edges labeled with -1 and $e_{f^{*}}(1)$ is the number of edges labeled with 1 . A graph $G$ is signed product cordial if it admits signed product cordial labeling.

Theorem 2.2: The Path graph $P_{n}, n \geq 2$ admits signed product cordial labeling.

Proof: Let $V=\left\{v_{1}, v_{2}, \ldots, v_{n}\right\}$ be the vertex set and $E=\left\{v_{i} v_{i+1} ; 1 \leq i \leq n-1\right\}$ be the edge set of the path graph 
$P_{n}$. To define vertex labeling $f: V(G) \rightarrow\{-1,1\}$ the following cases are to be considered.

Case 1 : when $n \equiv 0,1,3(\bmod 4)$

for $1 \leq i \leq n$

$$
f\left(v_{i}\right)=\left\{\begin{array}{r}
1 ; i \equiv 1,2 \bmod 4 \\
-1 ; i \equiv 0,3 \bmod 4
\end{array}\right.
$$

Case 2 : when $n \equiv 2(\bmod 4)$

for $1 \leq i \leq n-2$

$$
f\left(v_{i}\right)=\left\{\begin{array}{r}
1 ; i \equiv 1,2 \bmod 4 \\
-1 ; i \equiv 0,3 \bmod 4
\end{array}\right.
$$

and

$$
f\left(v_{n-1}\right)=1, f\left(v_{n}\right)=-1
$$

The induced edge labeling $f^{*}$ is given by

$$
\begin{aligned}
f^{*}\left(v_{i} v_{i+1}\right) & =f\left(v_{i}\right) f\left(v_{i+1}\right) \\
& = \begin{cases}1 & f\left(v_{i}\right) \text { and } f\left(v_{i+1}\right) \text { have same sign } \\
-1 & f\left(v_{i}\right) \text { and } f\left(v_{i+1}\right) \text { have different sign }\end{cases}
\end{aligned}
$$

with respect to the above labeling pattern we give the proof as follows,

i) When $n \equiv 0 \bmod 4$

The total number of vertices labeled with -1 's are given by $v_{f}(-1)=n / 2$ and the total number of vertices labeled with 1 's are given by $v_{f}(1)=n / 2$. Therefore the total difference between the vertices labeled with -1 's and 1 's is $\left|v_{f}(-1)-v_{f}(1)\right|=0$. The total number of edges labeled with -1 's are given by $e_{f^{*}}(-1)=(n / 2)-1$ and the total number of edges labeled with 1 's are given by $e_{f^{*}}(1)=(n / 2)$. Therefore the total difference between the edges labeled with -1 's and 1 's is

$$
\begin{aligned}
\left|e_{f^{*}}(-1)-e_{f^{*}}(1)\right| & =|-1|=1, \text { differ by one. } \\
& v_{f}(-1)=v_{f}(1)=n / 2 \\
& e_{f^{*}}(-1)+1=e_{f^{*}}(1)=n / 2
\end{aligned}
$$

ii) When $n \equiv 2 \bmod 4$

The total number of vertices labeled with -1 's are given by $v_{f}(-1)=n / 2$ and the total number of vertices labeled with 1 's are given by $v_{f}(1)=n / 2$. Therefore the total difference between the vertices labeled with -1 's and 1's is $\left|v_{f}(-1)-v_{f}(1)\right|=0$. The total number of edges labeled with -1 's are given by $e_{f^{*}}(-1)=(n / 2)$ and the total number of edges labeled with 1 's are given by $e_{f^{*}}(1)=(n / 2)-1$. Therefore the total difference between the edges labeled with -1 's and 1 's is $\left|e_{f^{*}}(-1)-e_{f^{*}}(1)\right|=1$, differ by one.

$$
\begin{aligned}
& v_{f}(-1)=v_{f}(1)=n / 2 \\
& e_{f^{*}}(-1)=e_{f^{*}}(1)+1=n / 2
\end{aligned}
$$

iii) When $n$ is odd
The total number of vertices labeled with -1 's are given by $v_{f}(-1)=(n-1) / 2$ and the total number of vertices labeled with 1 's are given by $v_{f}(1)=(n+1) / 2$. Therefore the total difference between the vertices labeled with -1 's and 1's is $\left|v_{f}(-1)-v_{f}(1)\right|=|-1|=1$. The total number of edges labeled with -1 's are given by $e_{f^{*}}(-1)=(n-1) / 2$ and the total number of edges labeled with 1 's are given by $e_{f^{*}}(1)=(n-1) / 2$. Therefore the total difference between the edges labeled with -1 's and 1 's is $\left|e_{f^{*}}(-1)-e_{f^{*}}(1)\right|=0$, differ by zero.

$$
\begin{aligned}
& v_{f}(-1)+1=v_{f}(1)=(n+1) / 2 \\
& e_{f^{*}}(-1)=e_{f^{*}}(1)=(n-1) / 2
\end{aligned}
$$

Thus in each cases we have $\left|v_{f}(-1)-v_{f}(1)\right| \leq 1$ and $\left|e_{f^{*}}(-1)-e_{f^{*}}(1)\right| \leq 1$. Hence the path graph $P_{n}, n \geq 2$ admits signed product cordial labeling.

Theorem 2.3: The Cycle graph $C_{n}, n \geq 3$ admits signed product cordial labeling except when $n \equiv 2 \bmod 4$.

Proof: Let $V=\left\{v_{1}, v_{2}, \cdots, v_{n}\right\}$ be the vertex set and $E=\left\{v_{i} v_{i+1} ; 1 \leq i \leq n-1\right\} \cup\left\{v_{1} v_{n}\right\}$ be the edge set of the cycle graph $C_{n}$. To define vertex labeling

$f: V(G) \rightarrow\{-1,1\}$ the following case is to be considered.

When $n \equiv 0,1,3(\bmod 4)$

for $1 \leq i \leq n$,

$$
f\left(v_{i}\right)=\left\{\begin{array}{l}
1 ; i \equiv 1,2 \bmod 4 \\
-1 ; i \equiv 0,3 \bmod 4
\end{array}\right.
$$

The edge labeling is given by

$$
\begin{aligned}
f^{*}\left(v_{i} v_{i+1}\right) & =f\left(v_{i}\right) f\left(v_{i+1}\right) \\
& =\left\{\begin{array}{cl}
1 & f\left(v_{i}\right) \text { and } f\left(v_{i+1}\right) \text { have same sign } \\
-1 & f\left(v_{i}\right) \text { and } f\left(v_{i+1}\right) \text { have different sign }
\end{array}\right.
\end{aligned}
$$

In view of the above labeling pattern we have, Table 1.

Table 1. Vertex and edge conditions of a cycle graph.

\begin{tabular}{cccc}
\hline$n$ & $v_{f}(-1)$ & $v_{f}(1)$ & $\left|v_{f}(-1)-v_{f}(1)\right|$ \\
\hline$n \equiv 0 \bmod 4$ & $n / 2$ & $n / 2$ & 0 \\
$n \equiv 1 \bmod 4$ & $(n-1) / 2$ & $(n+1) / 2$ & 1 \\
$n \equiv 3 \bmod 4$ & $(n-1) / 2$ & $(n+1) / 2$ & 1 \\
\hline \multicolumn{4}{c}{} \\
\hline$n \equiv 0 \bmod 4$ & $e_{f^{*}}(-1)$ & $e_{f^{*}}(1)$ & $\left|e_{f^{*}}(-1)-e_{f^{*}}(1)\right|$ \\
$n \equiv 1 \bmod 4$ & $(n-1) / 2$ & $n / 2$ & 0 \\
$n \equiv 3 \bmod 4$ & $(n+1) / 2$ & $(n-1) / 2$ & 1 \\
\hline
\end{tabular}


Hence the cycle graph $C_{n} ; n \neq 2 \bmod 4$ admits signed product cordial labeling.

Theorem 2.4: The star graph $K_{1, n}, n \geq 2$ admits signed product cordial labeling.

Proof: The star graph $K_{1, n}$ is a tree obtained by adding $n$ pendent edge to the center vertex. Let

$V=\left\{v_{1}, v_{2}, \cdots, v_{n}, v_{n+1}\right\}$ be the vertex set and the edge set is given by $E=\left\{v_{1} v_{i} ; 2 \leq i \leq n+1\right\}$. To define vertex labeling for $f: V(G) \rightarrow\{-1,1\}$ is given by for $1 \leq i \leq n+1$

$$
f\left(v_{i}\right)=\left\{\begin{array}{l}
1 ; i \equiv 1 \bmod 2 \\
-1 ; i \equiv 0 \bmod 2
\end{array}\right.
$$

When $n$ is even and odd, the edge labeling is given by

and

$$
\begin{aligned}
& f^{*}\left(v_{1} v_{2 i}\right)=-1 \\
& f^{*}\left(v_{1} v_{2 i+1}\right)=1 ; 1 \leq i \leq n / 2
\end{aligned}
$$

$$
\begin{aligned}
& f^{*}\left(v_{1} v_{2 i}\right)=-1 ; 1 \leq i \leq(n+1) / 2 \\
& f^{*}\left(v_{1} v_{2 i+1}\right)=1 ; 1 \leq i \leq((n+1) / 2)-1
\end{aligned}
$$

respectively.

In view of the above labeling pattern we have, Table 2.

Hence the star graph $K_{1, n}$ admits signed product cordial labeling.

\section{Signed Product Cordial Labeling for Special Graphs}

In this section we prove the signed product cordial labeling for the graphs $P_{n}^{+}, C_{n}^{+}$and the bistar graph $B_{n, n}$.

Theorem 3.1: The Path graph $P_{n}^{+}, n \geq 3$ admits signed product cordial labeling.

Proof: Let $v_{1}, v_{2}, v_{3} \cdots v_{n}$ and $u_{1}, u_{2}, u_{3} \cdots u_{n}$ be the vertex sets of the path graph $P_{n}^{+}$and the edge set is given by

$$
E_{1}=\left\{v_{i} v_{i+1} ; 1 \leq i \leq n-1\right\}, E_{2}=\left\{v_{i} u_{i} ; 1 \leq i \leq n\right\}
$$

The graph $P_{n}^{+}$has $2 n$ vertices and $2 n-1$ edges. To define vertex labeling $f: V(G) \rightarrow\{-1,1\}$ the following

Table 2. Vertex and edge conditions of a star graph.

\begin{tabular}{cccc}
\hline$n$ & $v_{f}(-1)$ & $v_{f}(1)$ & $\left|v_{f}(-1)-v_{f}(1)\right|$ \\
\hline$n \equiv 0 \bmod 2$ & $(n-1) / 2$ & $(n+1) / 2$ & 1 \\
$n \equiv 1 \bmod 2$ & $(n+1) / 2$ & $(n+1) / 2$ & 0 \\
\hline \multicolumn{4}{c}{} \\
\hline$n \equiv 0 \bmod 2$ & $e_{f^{*}}(-1)$ & $e_{f^{*}}(1)$ & $\left|e_{f^{*}}(-1)-e_{f^{*}}(1)\right|$ \\
$n \equiv 1 \bmod 2$ & $(n-1) / 2$ & $(n-3) / 2$ & 0 \\
\hline
\end{tabular}

cases are to be considered.

Case 1: when $n$ is even

for $1 \leq i \leq n$

$$
\begin{gathered}
f\left(v_{i}\right)=\left\{\begin{array}{l}
1 ; i \equiv 1,2 \bmod 4 \\
-1 ; i \equiv 0,3 \bmod 4
\end{array}\right. \\
f\left(u_{i}\right)=\left\{\begin{array}{l}
1 ; i \equiv 1 \bmod 2 \\
-1 ; i \equiv 0 \bmod 2
\end{array}\right.
\end{gathered}
$$

The edge labeling are defined as follows for $1 \leq i \leq n-1$

$$
\begin{aligned}
f^{*}\left(v_{i} v_{i+1}\right) & =f\left(v_{i}\right) f\left(v_{i+1}\right) \\
& = \begin{cases}1 & \text { if } f\left(v_{i}\right) \text { and } f\left(v_{i+1}\right) \text { have same sign } \\
-1 & \text { if } f\left(v_{i}\right) \text { and } f\left(v_{i+1}\right) \text { have different sign }\end{cases}
\end{aligned}
$$

for $1 \leq i \leq n$

$$
\begin{aligned}
f^{*}\left(v_{i} u_{i}\right) & =f\left(v_{i}\right) f\left(u_{i}\right) \\
& = \begin{cases}1 & \text { if } f\left(v_{i}\right) \text { and } f\left(v_{i+1}\right) \text { have same sign } \\
-1 & \text { if } f\left(v_{i}\right) \text { and } f\left(v_{i+1}\right) \text { have different sign }\end{cases}
\end{aligned}
$$

Case 2: When $n$ is odd

The vertex labeling is given by for $1 \leq i \leq n-2$

$$
\begin{gathered}
f\left(v_{i}\right)=\left\{\begin{array}{l}
1 ; i \equiv 1,2 \bmod 4 \\
-1 ; i \equiv 0,3 \bmod 4
\end{array}\right. \\
f\left(v_{n-1}\right)=1 ; f\left(v_{n}\right)=-1
\end{gathered}
$$

for $1 \leq i \leq n-1$

$$
\begin{aligned}
& f\left(u_{i}\right)=\left\{\begin{array}{l}
1 ; i \equiv 1 \bmod 2 \\
-1 ; i \equiv 0 \bmod 2
\end{array}\right. \\
& f\left(u_{n}\right)=-1
\end{aligned}
$$

The edge labeling are defined as follows for $1 \leq i \leq n-1$

$$
\begin{aligned}
f^{*}\left(v_{i} v_{i+1}\right) & =f\left(v_{i}\right) f\left(v_{i+1}\right) \\
& = \begin{cases}1 & \text { if } f\left(v_{i}\right) \text { and } f\left(v_{i+1}\right) \text { have same sign } \\
-1 & \text { if } f\left(v_{i}\right) \text { and } f\left(v_{i+1}\right) \text { have different sign }\end{cases}
\end{aligned}
$$

for $1 \leq i \leq n$

$$
\begin{aligned}
f^{*}\left(v_{i} u_{i}\right)= & f\left(v_{i}\right) f\left(u_{i}\right) \\
& = \begin{cases}1 & \text { if } f\left(v_{i}\right) \text { and } f\left(v_{i+1}\right) \text { have same sign } \\
-1 & \text { if } f\left(v_{i}\right) \text { and } f\left(v_{i+1}\right) \text { have different sign }\end{cases}
\end{aligned}
$$

In view of the above labeling pattern we have, Table 3.

Hence the graph $P_{n}^{+}, n \geq 3$ admits signed product cordial labeling.

Theorem 3.2: The graph $C_{n}^{+}, n \geq 3$ admits signed product cordial labeling except for $n \equiv 2 \bmod 4$. 
Table 3. Vertex and edge conditions of the graph $P_{n}^{+}, n \geq 3$.

\begin{tabular}{cccc}
\hline$n$ & $v_{f}(-1)$ & $v_{f^{\prime}}(1)$ & $\left|v_{f}(-1)-v_{f}(1)\right|$ \\
\hline$n \equiv 0 \bmod 2$ & $n$ & $n$ & 0 \\
$n \equiv 1 \bmod 4$ & $n$ & $n$ & 0 \\
$n \equiv 3 \bmod 4$ & $n$ & $n$ & 0 \\
\hline & & & \\
\hline$n$ & $e_{f^{*}}(-1)$ & $e_{f^{*}}(1)$ & $\left|e_{f^{*}}(-1)-e_{f^{*}}(1)\right|$ \\
\hline$n \equiv 0 \bmod 2$ & $n-1$ & $n$ & 1 \\
$n \equiv 1 \bmod 4$ & $n$ & $n-1$ & 1 \\
$n \equiv 3 \bmod 4$ & $n-1$ & $n$ & 1 \\
\hline
\end{tabular}

Proof: Let $v_{1}, v_{2}, v_{3} \cdots v_{n}$ and $u_{1}, u_{2}, u_{3} \cdots u_{n}$ be the vertex sets of the graph $C_{n}^{+}, n \geq 3$ with $2 n$ vertices and $2 n$ edges. The edge set is given by

$$
\begin{aligned}
& E_{1}=\left\{v_{i} v_{i+1} ; 1 \leq i \leq n-1\right\} \cup\left\{v_{1} v_{n}\right\} \\
& E_{2}=\left\{v_{i} u_{i} ; 1 \leq i \leq n\right\}
\end{aligned}
$$

The vertex labeling is defined by for $1 \leq i \leq n$

$$
\begin{aligned}
& f\left(v_{i}\right)=\left\{\begin{array}{l}
1 ; i \equiv 1,2 \bmod 4 \\
-1 ; i \equiv 0,3 \bmod 4
\end{array}\right. \\
& f\left(u_{i}\right)=\left\{\begin{array}{l}
-1 ; i \equiv 1 \bmod 2 \\
1 ; i \equiv 0 \bmod 2
\end{array}\right.
\end{aligned}
$$

The edge labeling is given by for $1 \leq i \leq n$

$$
\begin{aligned}
f^{*}\left(v_{i} v_{i+1}\right) & =f\left(v_{i}\right) f\left(v_{i+1}\right) \\
& = \begin{cases}1 & \text { if } f\left(v_{i}\right) \text { and } f\left(v_{i+1}\right) \text { have same sign } \\
-1 & \text { if } f\left(v_{i}\right) \text { and } f\left(v_{i+1}\right) \text { have different sign }\end{cases} \\
f^{*}\left(v_{1} v_{n}\right) & = \begin{cases}1 & \text { if } f\left(v_{i}\right) \text { and } f\left(v_{i+1}\right) \text { have same sign } \\
-1 & \text { if } f\left(v_{i}\right) \text { and } f\left(v_{i+1}\right) \text { have different sign }\end{cases} \\
f^{*}\left(v_{i} u_{i}\right) & = \begin{cases}1 & f\left(v_{i}\right) \text { and } f\left(v_{i+1}\right) \text { have same sign } \\
-1 & f\left(v_{i}\right) \text { and } f\left(v_{i+1}\right) \text { have different sign }\end{cases}
\end{aligned}
$$

In view of the above labeling pattern we have, the total number of vertices labeled with -1 's are given by $v_{f}(-1)=n$ and the total number of vertices labeled with 1 's are given by $v_{f}(1)=n$. Therefore the total difference between the vertices labeled with -1 's and 1's is $\left|v_{f}(-1)-v_{f}(1)\right|=0$. The total number of edges labeled with -1 's are given by $e_{f^{*}}(-1)=n$ and the total number of edges labeled with 1's are given by $e_{f^{*}}(1)=n$. Therefore the total difference between the edges labeled with -1 's and 1 's is $\left|e_{f^{*}}(-1)-e_{f^{*}}(1)\right|=0$, differ by zero.

$$
\begin{aligned}
& v_{f}(-1)=v_{f}(1)=n \\
& e_{f^{*}}(-1)=e_{f^{*}}(1)=n
\end{aligned}
$$

Hence the cycle graph $C_{n}^{+}, n \geq 3$ admits signed product cordial labeling.

Theorem 3.3: The graph $B_{n, n}, n \geq 2$ admits signed product cordial labeling.

Proof: The graph $B_{n, n}, n \geq 2$ is a bistar obtained from two disjoint copies of $K_{1, n}$ by joining the centre vertices by an edge. It has $2 n+2$ vertices and $2 n+1$ edges. Let $v_{1}, v_{2}, v_{3}, \cdots, v_{2 n+2}$ be the vertices of the bistar graph. The edge set is defined as

$$
\begin{aligned}
& E_{1}=\left\{v_{1} v_{2 i+1} ; 1 \leq i \leq n\right\}, \\
& E_{2}=\left\{v_{2} v_{2 i} ; 2 \leq i \leq n+1\right\}
\end{aligned}
$$

We now define vertex labeling $f: V(G) \rightarrow\{-1,1\}$ as

$$
\begin{aligned}
& f\left(v_{i}\right)=\left\{\begin{array}{r}
1 ; i \equiv 1 \bmod 2 ; 1 \leq i \leq 2 n \\
-1 ; i \equiv 0 \bmod 2 ; 3 \leq i \leq 2 n+2
\end{array}\right. \\
& f\left(v_{2}\right)=1 \\
& f\left(v_{2 n+1}\right)=-1
\end{aligned}
$$

The edge labeling is given by

$$
\begin{aligned}
& f\left(v_{2} v_{2 i}\right)=-1 ; 2 \leq i \leq n+1 \\
& f\left(v_{1} v_{2 n+1}\right)=-1 \\
& f\left(v_{1} v_{2 i+1}\right)=1 ; 1 \leq i \leq n-1
\end{aligned}
$$

The total number of vertices labeled with -1 's are given by $v_{f}(-1)=n+1$ and the total number of vertices labeled with 1 's are given by $v_{f}(1)=n+1$. Therefore the total difference between the vertices labeled with -1 's and 1 's is $\left|v_{f}(-1)-v_{f}(1)\right|=0$. The total number of edges labeled with -1 's are given by $e_{f^{*}}(-1)=n$ and the total number of edges labeled with 1 's are given by $e_{f^{*}}(1)=n+1$. Therefore the total difference between the edges labeled with -1 's and 1's is $\left|e_{f^{*}}(-1)-e_{f^{*}}(1)\right|=1$, differ by one. In view of the above labeling pattern we have,

$$
\begin{aligned}
& v_{f}(-1)=v_{f}(1)-1=n+1 \\
& e_{f^{*}}(-1)=e_{f^{*}}(1)+1=n+1
\end{aligned}
$$

From the above labeling pattern we have $\left|v_{f}(-1)-v_{f}(1)\right| \leq 1$ and $\left|e_{f^{*}}(-1)-e_{f^{*}}(1)\right| \leq 1$. Hence the bistar graph $B_{n, n}, n \geq 2$, admits signed product cordial labeling.

Example 3.4: Figure 1 illustrates the signed product cordial labeling for Bistar $B_{5,5}$. Among the eleven edges five edges receive the label +1 and six edges receive the label -1 . 


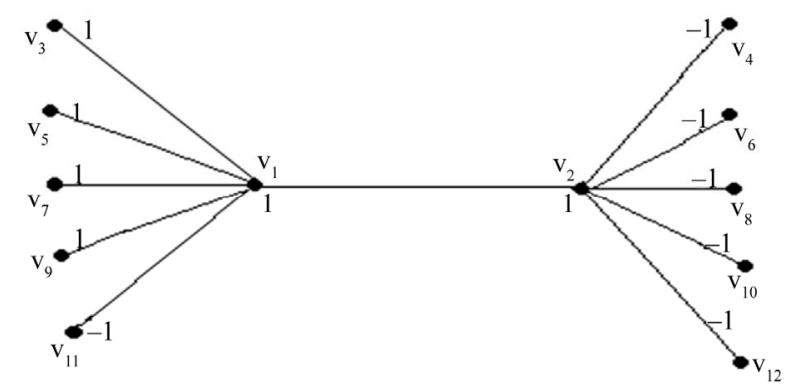

Figure 1. Signed product cordial labeling of Bistar $\boldsymbol{B}_{5,5}$.

\section{General Results on Signed Product Cordial Labeling}

In this section we prove the Signed product cordial labeling for the some general graphs.

Definition 4.1: The tree $T @ m K_{1}$ is obtained by attaching $m$ copies of $K_{1}$ to any one of the vertices in $T$.

Theorem 4.2: If a tree $T$ admits signed product cordial labeling with $n$ vertices then $T @ m K_{1}$ ( $m$ even) is also a signed product cordial tree.

Proof: Let us assume that a tree $T$ admits signed product cordial labeling. The mapping $f: V \rightarrow\{-1,1\}$ satisfies the condition of signed product cordial labeling.

Let $T^{\prime}=T @ m K_{1}=\left(V^{\prime}, E^{\prime}\right)$ where $V^{\prime}=V \cup\left\{u_{1}, u_{2}, \cdots, u_{n}\right\}$ and $E^{\prime}=E \cup\left\{v u_{1}, v u_{2}, \cdots, v u_{n}\right\}$. The vertex labeling for $T^{\prime}, g: V^{\prime} \cup\{-1,1\}$ is defined as $g(v)=f(v) \forall v \in V$.

Let $\left\{u_{1}, u_{2}, \cdots, u_{m}\right\}$ be the $m$ vertices joined to the vertex $v$ of the tree $T$. The vertex labeling of $\left\{u_{1}, u_{2}, \cdots, u_{m}\right\}$ is given by $g\left(u_{i}\right)=(-1)^{i} ; 1 \leq i \leq m$. If $f(v)=1$, then the sign of $g\left(v u_{1}\right), g\left(v u_{2}\right), \cdots, g\left(v u_{m}\right)$ will be the sign of $g\left(u_{1}\right), g\left(u_{2}\right), \cdots, g\left(u_{m}\right)$ respectively and if $f(v)=-1$ then the sign of $g\left(v u_{1}\right), g\left(v u_{2}\right), \cdots, g\left(v u_{m}\right)$ will be the sign of $-g\left(u_{1}\right),-g\left(u_{2}\right), \cdots,-g\left(u_{m}\right)$. In both the cases the new $m$ edges $\left\{v u_{1}, v u_{2}, \cdots, v u_{m}\right\}$ contributes equal number of edges labeled with -1 's and 1 's. Therefore in a tree $T @ m K_{1}$, the difference between the total number of vertices labeled with -1 's and 1's and the difference between the total number of edges labeled with -1 's and 1's differs by utmost one.

Example 4.3: Figure 2 illustrates the signed product cordial labeling for $T @ 4 K_{1}$ where $T$ is an arbitrary tree having signed product cordial labeling.

Corollary 4.4: If a connected graph $G$ has signed product cordial labeling then the graph $G @ m K_{1}$ where $m$ is even admits signed product cordial labeling.

Definition 4.5: The tree $T \hat{0} P_{n}$ is obtained by superimposing a pendant vertex of $P_{n}$ with any of the selected vertex in $T$.

Theorem 4.6: If a tree $T$ as signed product cordial labeling then $T \hat{0} P_{n}$ where $m$ is odd admits signed product cordial labeling.

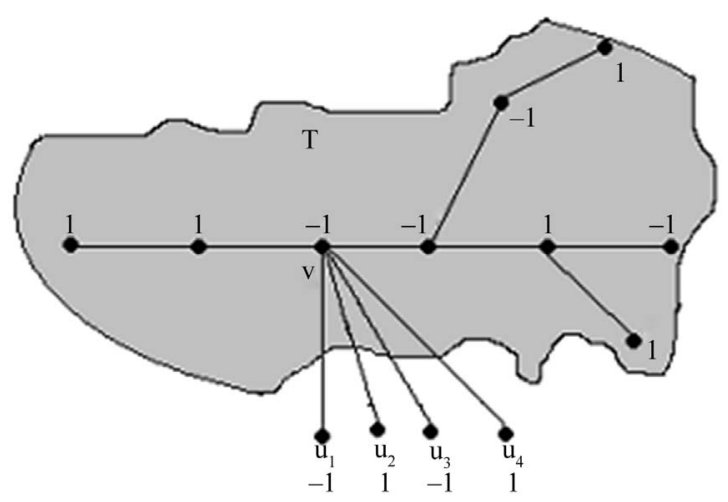

Figure 2. Signed product cordial labeling of $T @ 4 K_{1}$.

Proof: Let $T=(V, E)$ be a connected graph with vertex set $V$ and edge set $E$ then the tree $T \hat{0} P_{n}=\left(V^{\prime}, E^{\prime}\right)$ where $V^{\prime}=V \cup\left\{u_{1}, u_{2}, \cdots, u_{n}\right\}$ and $E^{\prime}=E \cup\left\{u_{i} u_{i+1} ; 1 \leq i \leq n-1\right\}$. Let $v$ be a vertex in $T$. we superimpose a pendant vertex with a selected vertex $v$ in $T$ only if they preserves the same sign.

Case 1: If $f(v)=f\left(u_{1}\right)=1$, then the vertex labeling for the path graph follows from theorem 2.2

Case 2: If $f(v)=f\left(u_{1}\right)=-1$, then the vertex labeling is given by

Sub Case 2.1: when $n \equiv 0,1,3(\bmod 4)$ for $1 \leq i \leq n$

$$
\begin{aligned}
f\left(v_{i}\right)= & 1 ; i \equiv 0,3 \bmod 4 \\
& =-1 ; i \equiv 1,2 \bmod 4
\end{aligned}
$$

Sub Case 2.2: when $n \equiv 2(\bmod 4)$ for $1 \leq i \leq n-2$

$$
\begin{aligned}
f\left(v_{i}\right)=1 ; i \equiv 0,3 \bmod 4 \\
=-1 ; i \equiv 1,2 \bmod 4
\end{aligned}
$$

and

$$
f\left(v_{n-1}\right)=-1, f\left(v_{n}\right)=1
$$

As we superimpose the pendant vertex of $P_{n}$ with any one of the vertex in $T$ provided they preserve the same sign then the path graph contributes equal number of vertices and edges labeled with -1 's and 1's. Hence the tree $T \hat{0} P_{n}$ admits signed product cordial labeling.

Corollary 4.7: If a connected graph $G$ has signed product cordial labeling then the graph $T \hat{0} P_{n}$ where $n$ is odd admits signed product cordial labeling.

Theorem 4.8: If a connected graph $G$ has a signed product cordial labeling with $n$ vertices $(n \equiv 0 \bmod 4)$ then $\mathrm{G}^{+}$admits signed product cordial.

Proof: Let $G=(V, E)$ be a connected graph with vertex set $V=\left\{u_{1}, u_{2}, \cdots, u_{n}\right\}$. Since $G$ has a signed product cordial labeling there exists $f: V \rightarrow\{-1,1\}$ such that $\left|v_{f}(-1)-v_{f}(1)\right| \leq 1$ and $\left|e_{f^{*}}(-1)-e_{f^{*}}(1)\right| \leq 1$. 
As $n \equiv 0 \bmod 4,\left|v_{f}(-1)-v_{f}(1)\right|=0$. Let $V=\left\{V_{1} \cup V_{2}\right\}$ where $V_{1}=\left\{u_{i}: i \equiv 1 \bmod 2\right\}$ and $V_{2}=\left\{u_{i}: i \equiv 0 \bmod 2\right\}$. For our convenience let us assume that $f$ maps all the vertices of $V_{1}$ to 1 and all the vertices of $V_{2}$ to -1 . The graph $G^{+}=\left(V^{\prime}, E^{\prime}\right)$ is obtained by attaching the pendant vertices $\left\{u_{1}^{\prime}, u_{2}^{\prime}, \cdots, u_{n}^{\prime}\right\}$ to each of the vertices $\left\{u_{1}, u_{2}, \cdots, u_{n}\right\}$ in G. Let the vertex set and edge set of $G^{+}$be defined as $V^{\prime}=\left\{u_{i}, u_{i}^{\prime}: 1 \leq i \leq n\right\}$ and $E^{\prime}=E \cup\left\{u_{i} u_{i}^{\prime} ; 1 \leq i \leq n\right\}$.

The vertex labeling for the graph $G^{+}, g: V^{\prime} \rightarrow\{-1,1\}$ is defined as follows for, $1 \leq i \leq n$

$$
g\left(u_{i}\right)=f\left(u_{i}\right)
$$

$$
\begin{aligned}
& g\left(u_{i}^{\prime}\right) \\
& =\left\{\begin{aligned}
&-1 \text { when } i \equiv 0 \bmod 2 \text { and } f\left(u_{i}\right)=-1 \text { if } i \equiv 0 \bmod 4 \\
& 1 \text { when } i \equiv 1 \bmod 2 \text { and } f\left(u_{i}\right)=1 \text { if } i \equiv 1 \bmod 4 \\
&-1 \text { when } i \equiv 0 \bmod 2 \text { and } f\left(u_{i}\right)=1 \text { if } i \equiv 2 \bmod 4 \\
& 1 \text { when } i \equiv 1 \bmod 2 \text { and } f\left(u_{i}\right)=-1 \text { if } i \equiv 3 \bmod 4
\end{aligned}\right.
\end{aligned}
$$

The induced edge labels $g^{*}(u v)=f(u v) \forall u v \in E$. All the newly added edges $g^{*}\left(u_{i} u_{i}^{\prime}\right)$ will share equally the labels -1 and 1 as per our construction above. Hence $\left|v_{g}(-1)-v_{g}(1)\right|=0$ and $\left|e_{g^{*}}(-1)-e_{g^{*}}(1)\right|=0$. Only by this labeling pattern the graph $G^{+}$admits signed product labeling where $n$ is a multiple of 4 .

\section{Acknowledgements}

The referee is gratefully acknowledged for their suggestions that improved the manuscript.

\section{References}

[1] I. Cahit, "Cordial Graphs: A Weaker Version of Graceful and Harmonious Graphs," Ars Combinatoria, Vol. 23, 1987, pp. 201-207.

[2] M. Sundaram, R. Ponraj and S. Somasundram, "Total Product Cordial Labeling of Graphs," Bulletin of Pure and Applied Sciences: Section E. Mathematics and Statistics, Vol. 25, 2006, pp. 199-203.

[3] J. A. Gallian, "A Dynamic Survey of Graph Labeling," Electronic Journal of Combinatorics, Vol. 17, No. DS6, 2010, pp. 1-246.

[4] S. K. Vaidya, N. A. Dani, K. K. Kanani and P. L. Vihol, "Cordial and 3-Equitable Labeling for Some Star Related Graphs," International Mathematical Forum, Vol. 4, No. 31, 2009, pp. 1543-1553. 